\title{
Circulating Interferon-gamma and White Matter Brain Damage in Preterm Infants
}

\author{
INGRID HANSEN-PUPP, SOLVEIG HARLING, ANN-CATHRINE BERG, CORRADO CILIO, \\ LENA HELLSTRÖM-WESTAS, AND DAVID LEY
}

\author{
Department of Pediatrics [I.H.-P., S.H., A.-C.B., L.H.-W., D.L.], Lund University Hospital, 22185 Lund, \\ Sweden, Department of Clinical Sciences and Department of Pediatrics, [C.C.], Cellular Autoimmunity \\ Unit, Wallenberg Laboratory, Malmoe University Hospital, 20502 Malmoe, Sweden
}

\begin{abstract}
The fetal inflammatory response has been suggested as causal in neonatal morbidity. Serial levels of circulating cytokines were evaluated in 74 infants with a mean gestational age (GA) of 27.1 wk. Pro-inflammatory and modulatory (IL-4, IL-10) cytokines were analyzed from cord blood, and at 6,24 , and $72 \mathrm{~h}$ postnatal age. Measure of cytokine burden over time was assessed by calculating the area under curve (AUC) for analyzed levels $(0-72 \mathrm{~h})$. Premature rupture of membranes (PROM) was associated with higher levels of IL- 2 at birth and at $6 \mathrm{~h}$, of IFN- $\gamma$ at 6 and $24 \mathrm{~h}$ postnatal age and of TNF- $\alpha$ at 6 and $24 \mathrm{~h}$. Levels of IFN- $\gamma$ at 6,24 , and $72 \mathrm{~h}$ were increased in infants developing white matter brain damage (WMD) compared with those without WMD. Infants with arterial hypotension requiring dopamine treatment had an increase in IL-6 with a peak at $6 \mathrm{~h}$ of age. Severe intraventricular hemorrhage (IVH) was associated with increase in AUC, whereas WMD was associated with increase in AUC. A fetal immune response with increased postnatal levels of IFN- $\gamma$ was associated with development of WMD. PROM was
\end{abstract}

\section{ABSTRACT}

associated with a T-helper 1 cytokine response with increased levels of IFN- $\gamma$. Type of inflammatory response appears of importance for subsequent morbidity. (Pediatr Res 58: 946-952, 2005)

Abbreviations
CI, area under curve
GA, gestational age
IFN- $\gamma$, interferon- $\gamma$
IVH, intraventricular hemorrhage
MABP, mean arterial blood pressure
OR, odds ratio
PROM, premature rupture of membranes
PVL, periventricular leukomalacia
Th, T-helper
TNF- $\alpha$, tumor necrosis factor- $\alpha$
WMD, white matter brain damage

Antenatal infection is considered to be a major cause of preterm birth and elicits a fetal inflammatory response that has been suggested to be causal in both early acute neonatal morbidity and in chronic neurologic morbidity $(1,2)$. Preterm labor and rupture of membranes, histologic chorioamnionitis, and funisitis have been associated with early neonatal brain injury and cerebral palsy (CP) (3-6). Elevated levels of proinflammatory cytokines in amniotic fluid, fetal and umbilical cord blood, and in postnatal blood during the first $12 \mathrm{~h}$ have been correlated to hemodynamic impairment, IVH, WMD, and CP (2,5,7-9). On the other hand, a recent study failed to show

Received January 6, 2005; accepted March 21, 2005.

Correspondence: Ingrid Hansen-Pupp, M.D., Department of Pediatrics, Lund University Hospital, 22185 Lund, Sweden; e-mail ingrid.pupp@skane.se

This study was supported by the Swedish Medical Research Council (grants 14940, 4732, and 6535 to C.M.C.), Lunds University Hospital and Lund Medical Faculty grants, the Gorthon Foundation and the Nils W. Svenningsen Foundation for Preterm Infants. C.M.C. was funded by grants from JDRF (\#11-2004-38) and Skåne Research Council (1036/03; 709/02).

DOI: 10.1203/01.PDR.0000182592.76702.E8 any association between postnatal circulating cytokines at a median age of $2.4 \mathrm{~d}$ and development of $\mathrm{CP}$ in preterm infants (10).

Induced inflammation in humans results in rapid changes in circulating levels of cytokines with a half-life varying between different cytokines (11). A study with postnatal serial sampling inferred that levels of several circulating pro-inflammatory cytokines were highest after birth and subsequently declined in a small group of preterm infants indicating that cytokine levels in neonatal blood may be very different from levels in umbilical cord blood $(12,13)$. This may explain the lack of association between postnatal circulating cytokines and development of CP in the study by Nelson et al. (10). The process of birth, associated with stress and major physiologic changes, may have a profound effect on the circulating inflammatory response in the preterm infant. Therefore, a serial assessment of circulating cytokine levels at defined time points, incorporating the transition from fetal to neonatal life, may improve understanding of the association between systemic inflammatory response and morbidity in preterm infants. 
The theory of a preferential Th1 or a Th2 differentiation with a subsequent Th1 (TNF- $\alpha$, IFN- $\gamma$, IL-2, IL-12) or a Th2 (IL-4, IL-6, IL-10) cytokine response being implicated in tissue damage or protection has been explored in several different areas of disease (14). Genetic and environmental factors, e.g. properties of inducing microbial compounds may influence the Th1/Th2 differentiation resulting in different patterns of cytokine response (14). PROM is a condition with a high combined prevalence of intra-amniotic inflammation and infection and has been associated with increased levels of Th1 cytokines in umbilical and maternal blood (15-17). The Th1/Th2 paradigm may prove useful for distinguishing mechanistic links between the fetal inflammatory response and postnatal morbidity in preterm infants.

The aim of this study was to evaluate circulating levels of pro-inflammatory and modulatory cytokine levels in umbilical cord blood and in neonatal blood at 6, 24, and $72 \mathrm{~h}$ of postnatal age and determine their relationship to PROM as a marker of antenatal inflammation, early hemodynamic changes, and morphologic brain damage in preterm infants. We aimed to evaluate changes in cytokine levels over time as differences between defined time points and to perform an integrated assessment of cytokine burden over time.

\section{METHODS}

Study population and clinical routines. The study was a 2-y (February 2001 to February 2003) prospective cohort study of infants born at Lund University Hospital. The study was approved by the Regional Committee for Research Ethics at Lund University.

Pregnant women with a risk of delivery before $32 \mathrm{wk}$ were identified and included before delivery after written informed consent from both parents. The acceptance rate for participation in the study was $86 \%$. Inclusion criteria were a GA $<32$ wk at birth, antenatal informed consent, and absence of major congenital anomalies. All pregnancies were dated by ultrasound at 17-18 gestational weeks. Seventy-four infants were enrolled in the study. After delivery, the infants were admitted to the Neonatal Intensive Care Unit, Lund University Hospital.

An umbilical or peripheral arterial catheter was inserted within $1 \mathrm{~h}$ after birth for blood sampling, including blood culture and for continuous bloodpressure monitoring (M3153A, Viridia Surveillance Center, Hewlett-Packard, Palo Alto, CA). Indication for treatment of arterial hypotension was a MABP $(\mathrm{mm} \mathrm{Hg})$ lower than the infants' GA in weeks during the first 3 postnatal days. Treatment of arterial hypotension included intravenous infusion of dopamine in a starting dose of $3-5 \mu \mathrm{g} / \mathrm{kg} / \mathrm{min}$ with a subsequent increase up to maximum of $15 \mu \mathrm{g} / \mathrm{kg} / \mathrm{min}$ and/or volume expansion (fresh frozen plasma, 5\% albumin, $0.9 \%$ sodium hydrochloride, or packed erythrocytes if hemoglobin levels were below $140 \mathrm{~g} / \mathrm{L}$ ) until MABP had reached acceptable levels and the infant had sufficient diuresis, i.e. a urinary output of $\geq 2 \mathrm{~mL} / \mathrm{kg} / \mathrm{h}$.

Quantitative analysis of plasma cytokines. Sampling was performed from umbilical cord blood and from arterial blood at 6,24 , and $72 \mathrm{~h}$ through the indwelling arterial line. Blood samples were collected in a Vacutainer tube containing the anticoagulant EDTA (BD Biosciences, San Jose, CA), put on ice, and delivered within 20 min to the local chemical laboratory where the plasma was separated into several aliquots and then stored in a freezer $\left(-70^{\circ} \mathrm{C}\right)$ until analyzed in one batch at $7 \mathrm{mo}$ after termination of the study. Levels of pro-inflammatory (TNF- $\alpha$, IFN- $\gamma$, IL-1 $\beta$, IL-2, IL-6, IL-8, IL-12) and modulatory (IL-4, IL-10) cytokines in plasma were determined by cytometric bead array (CBA; BD Biosciences) and flow cytometry according to the manufacturer's recommendations. This assay is based on a mixture of six microbead populations with distinct fluorescent intensities (FL-3) precoated with capture antibodies specific for each cytokine and uses the sensitivity of fluorescence detection by flow cytometry to measure soluble cytokines in a particle-based immunoassay. Each bead provides a capture surface for a specific cytokine and is analogous to an individually coated well in an ELISA plate. Briefly, $50 \mu \mathrm{L}$ of mixed beads coated with cytokine-specific capture antibodies were added to $50 \mu \mathrm{L}$ of patient plasma and incubated for $1.5 \mathrm{~h}$ at room temperature. After washing, $50 \mu \mathrm{L}$ of phycoerythrin-conjugated (PE) anti-human inflammatory cytokine antibodies were added. Simultaneously, 50 $\mu \mathrm{L}$ of standards for each cytokine $(0-5000 \mathrm{pg} / \mathrm{mL})$ were treated likewise to generate a standard curves. Two-color flow cytometric analysis was performed using a FACSCalibur flow cytometer (BD Biosciences). Data were acquired and analyzed using BD Biosciences CBA software. Forward- versus sidescatter gating was used to exclude any sample particles other than the 7.5- $\mu \mathrm{m}$ polystyrene beads. Flow cytometric analysis was performed and analyzed by a single operator and cytokine concentrations were determined based on the standard curves using the CBA software. The lower limit of detection for the various cytokines evaluated ranged from 2 to $10 \mathrm{pg} / \mathrm{mL}$. For results above the upper limit of detection, serial dilution of the sample was performed to accurately determine cytokine levels. A level of $\leq 0.1 \mathrm{pg} / \mathrm{mL}$ was regarded as nondetectable.

Hemodynamic evaluation. Values of mean, systolic, and diastolic arterial blood pressure, oxygen saturation, and heart rate were digitally stored every 15 min during the first $72 \mathrm{~h}$. In the infants without an indwelling arterial line $(n$ $=9$ ), noninvasive blood pressure (neonatal blood pressure cuff, Hewlett Packard) was obtained every $60 \mathrm{~min}$ and documented in the protocol. Administered treatment with dopamine was prospectively registered during the first $72 \mathrm{~h}$ of life.

Cranial ultrasonography. Repeated ultrasound examinations of the brain were performed by two of the investigators (I.H.-P., D.L.) using an Acuson XP $512(7.5 \mathrm{MHz})$ or Acuson Sequoia (8.5 MHz) (Mountain View, CA) at 1, 3, and 7 postnatal days, at $6 \mathrm{wk}$, and at 40 gestational weeks. Data from the ultrasound brain examinations were stored digitally and forwarded to the Department of Pediatric Radiology, where the images were reviewed by a pediatric radiologist. Images with suspected abnormalities where reassessed by a pediatric radiologist blinded to the clinical history. Severe IVH was defined in the presence of IVH grade 3 and/or parenchymal hemorrhagic infarction. WMD was defined in the presence of periventricular echodensities persisting for more than $7 \mathrm{~d}$ or periventricular cysts (18).

Clinical data. Antenatal data were obtained from maternal records. Preeclampsia was defined as blood pressure $\geq 140 / 90 \mathrm{~mm} \mathrm{Hg}$ and albuminuria $>0.3 \mathrm{~g} / \mathrm{L} / \mathrm{d}$, and PROM as a rupture of membranes before the onset of labor. Suspected maternal infection was defined in the case of elevated maternal C-reactive protein $>5 \mathrm{mg} / \mathrm{L}$ and/or fever $>38^{\circ} \mathrm{C}$. Clinical chorioamnionitis was defined when two of the following criteria were present: maternal fever $>38^{\circ} \mathrm{C}$, maternal tachycardia, fetal tachycardia, malodorous amniotic fluid, and uterine tenderness. The total number of doses of antenatal steroids and the time point of the last dose before delivery were registered. The infants were defined as small for gestational age (SGA) if the deviation of birth weight (BW) was $>2$ SD below the gestational age-related mean of the population (19). Neonatal data were obtained from the infants' hospital records until home discharge. All infants remained in the tertiary level NICU in Lund for more than $72 \mathrm{~h}$

Statistical analysis. Statistical analysis was performed using SPSS v 11.5 for Microsoft Windows (SPSS Inc., Chicago, IL). Plasma levels of the respective cytokines from umbilical cord and at 6,24 , and $72 \mathrm{~h}$ of postnatal age were used to calculate an AUC as an assessment of cytokine burden over time in each subject. AUC was calculated according to the trapezium rule (20). AUC was only calculated in subjects with three or more valid plasma samples (59 subjects with 4 samples, 10 subjects with 3 samples; $n=69$ ). Calculated AUC was adjusted for total sampling period, being either 66 or $72 \mathrm{~h}$, thus achieving a weighted average level over time. Levels of cytokine levels at the respective sampling points as well as the calculated AUC were logarithmically transformed to obtain a normal distribution of values. Average MABP $(0-72$ h) was calculated as the mean of aggregated data for each subject. Relationships between antenatal variables and cytokine levels with adjustment for other variables was assessed by using multiple regression analysis. Univariate analysis of cytokine levels at specific time points were assessed using the Kruskal-Wallis test for groups of uneven size. Relationships between cytokine levels and categorical or continuous outcome variables were assessed using logistic regression analysis (backward log-likelihood ratio) or multiple linear regression analysis as appropriate with adjustment for gestational age and gender. Correlations between cytokines were assessed with the Spearman rank correlation coefficient and differences between paired samples by the Wilcoxon rank sum test.

\section{RESULTS}

Clinical features of the study population. All mothers received antenatal steroid treatment. Of the 74 infants, 30 (40\%) had mothers with PROM occurring at a median (range) of 8 (1-72) d before delivery, 18 (24\%) were delivered due to maternal preeclampsia, $22(30 \%)$ had mothers with suspected maternal infection, 5 (7\%) had mothers with clinical chorio- 
amnionitis, and $53(72 \%)$ had mothers who had received antenatal antibiotic treatment. Fifty-two of the infants (70\%) were delivered by cesarean section and $34(46 \%)$ were either twins or triplets.

The 74 infants had a mean [SD (range)] gestational age at birth of 27 [2.0 (23-31)] wk with a mean (SD) birth weight of $1007(280) \mathrm{g}$. Thirty-nine $(53 \%)$ of the infants were males and $20(27 \%)$ had a birth weight SGA. Twenty-five (34\%) infants received dopamine treatment and $40(54 \%)$ volume expansion during the first $72 \mathrm{~h}$ of life. Ultrasound examinations according to the study protocol showed that 17 (23\%) infants developed any IVH, 6 (8\%) severe IVH, and 8 (11\%) WMD. Only one infant had a positive blood culture at birth.

Three infants died, one infant after $4 \mathrm{~h}$, one infant after $24 \mathrm{~d}$ (both with Escherichia coli infection) and one infant after $2 \mathrm{~d}$ (persistent pulmonary hypertension and parenchymal cerebral hemorrhage).

PROM and cytokines. Levels of cytokines at the respective times of sampling were analyzed in infants with and without maternal PROM. Table 1 demonstrates the antenatal characteristics and postnatal outcome according to presence or absence of PROM. WMD was more frequent in surviving infants after PROM compared with in those without PROM. The three infants who died were all delivered after PROM.

Infants delivered after PROM $(n=30)$ had higher levels of IL-2 at birth and at $6 \mathrm{~h}(p=0.012$ and $p=0.004)$, of IFN- $\gamma$ at 6 and $24 \mathrm{~h}$ postnatal age $(p=0.021$ and $p=0.011)$, of TNF- $\alpha$ at 6 and $24 \mathrm{~h}(p=0.014$ and $p=0.041)$, and AUC $(\mathrm{IFN}-\gamma)(p=0.037)$ compared with infants not delivered after PROM $(n=44)$. Levels of other cytokines did not differ significantly at the respective sampling points in relation to PROM although levels of IL-6 exhibited differences when analyzed over time within the respective groups. In infants delivered after PROM, median level of IL-6 was highest at birth and decreased to a lowest median value at $72 \mathrm{~h}(p=$ $0.033)$ compared with infants not delivered after PROM who exhibited a significant increase from birth to $6 \mathrm{~h}$ of age $(p=$ $0.022)$ with a subsequent decrease at $72 \mathrm{~h}(p=0.039)$. Median

Table 1. Antenatal characteristics and postnatal outcome according to presence or absence of premature rupture of membranes.

\begin{tabular}{lrlrlr}
\hline & $\begin{array}{c}\text { PROM } \\
(\mathrm{N}=30)\end{array}$ & $\begin{array}{r}\text { Non PROM } \\
(\mathrm{N}=44)\end{array}$ & P value \\
\hline Gender (male) & 18 & $(60)$ & 21 & $(48)$ & 0.299 \\
Chorioamnionitis & 4 & $(13)$ & 1 & $(2)$ & 0.151 \\
Maternal antibiotic treatment & 30 & $(100)$ & 23 & $(52)$ & $<0.001$ \\
Suspected maternal infection & 13 & $(43)$ & 9 & $(20)$ & 0.035 \\
Preeclampsia & 0 & $(0)$ & 18 & $(41)$ & $<0.001$ \\
Preterm labor & 20 & $(67)$ & 9 & $(20)$ & $<0.01$ \\
Gestational age (weeks) & 27.0 & $(1.7)$ & $27.2(2.1)$ & 0.753 \\
Birth weight (g) & 1070 & $(260)$ & 965 & $(289)$ & 0.112 \\
SGA & 1 & $(3)$ & 19 & $(43)$ & $<0.001$ \\
Dopamine treatment & 8 & $(27)$ & 17 & $(39)$ & 0.382 \\
White matter brain damage & 6 & $(22)$ & 2 & $(4)$ & $0.049 *$ \\
IVH (grade III/IV) & 3 & $(11)$ & 3 & $(7)$ & 0.676 \\
Died & 3 & $(10)$ & 0 & $(0)$ & 0.063 \\
\hline
\end{tabular}

Value expressed as mean (SD) or number (percentage). PROM, premature rupture of membranes; SGA, small for gestational age; IVH, intraventricular hemorrhage;* statistical difference calculated on surviving infants. levels and distribution of IFN- $\gamma$, IL-2, TNF- $\alpha$, and IL-6 according to presence or absence of PROM are illustrated in Figure 1.

Median levels of IL-6, IL-8, and IL-10 were significantly decreased by $72 \mathrm{~h}$ compared with levels during the first $24 \mathrm{~h}$ of life in infants with and in those without maternal PROM $(p<$ 0.01 for both). Median levels of IL-1 $\beta$, IL-12, and IL-4 remained unchanged from birth and up to $72 \mathrm{~h}$ in both groups. Levels of TNF- $\alpha$ and IL- $1 \beta$ were overall low with a few exceptions and with measured levels frequently below the detection level of the assays at all studied time points.

Levels of the modulatory cytokine IL-10 changed similarly between the studied time points in studied infants as is illustrated for each infant in Figure 2. Median level of IL-10 increased from birth to that at $6 \mathrm{~h}$ of age $(p<0.001)$, followed by a decrease between 6 and $24 \mathrm{~h}$ and between 24 and $72 \mathrm{~h}(p$ $<0.001$ and $p=0.002$, respectively).

Correlations were analyzed between AUC of measured cytokines. Three groups of cytokines were identified with positive correlations between the AUC of the cytokines within the respective groups. The respective groups were: 1) IL-6, IL-8, and IL-10 ( $r=0.49-0.68) ; 2)$ IFN- $\gamma$, IL-1 $\beta$, and IL-12 $(r=$ $0.34-0.69)$; and 3) TNF- $\alpha$, IL-4, and IL-2 ( $r=0.38-0.52)$. IL-10 also exhibited significant correlations to IFN- $\gamma$ and IL-4 ( $r=0.4$ for both). All described correlations with $p<0.01$. No inverse correlations were observed between AUC of measured cytokines.

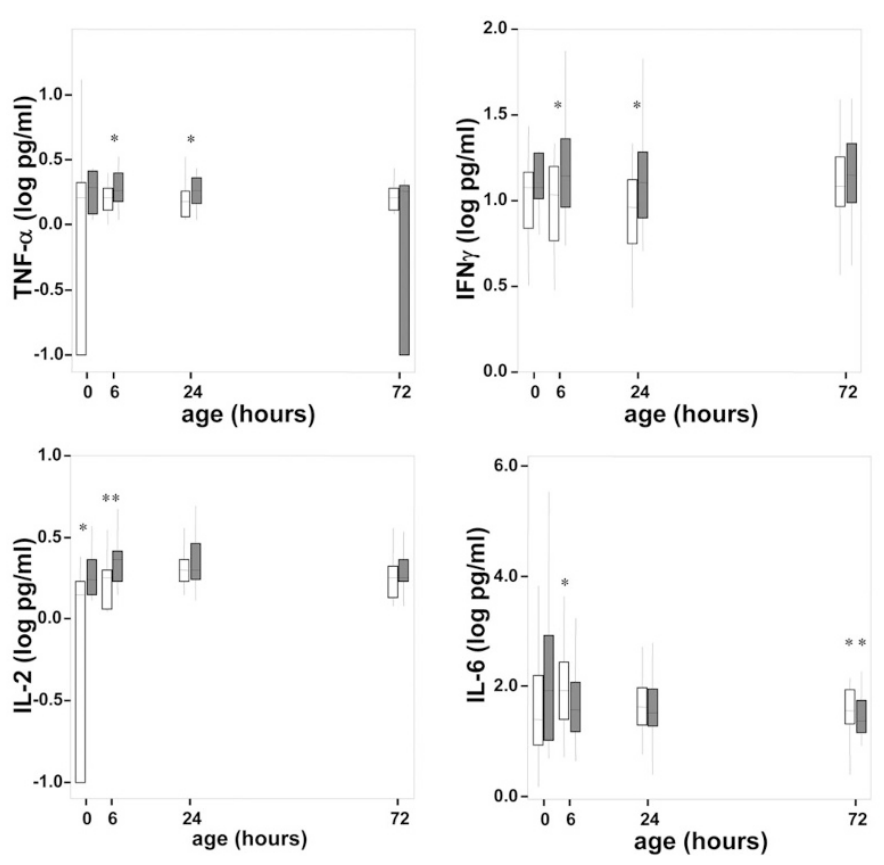

Figure 1. Plasma levels of IFN- $\gamma$, IL-2, TNF- $\alpha$, and IL-6 (log pg/mL) at birth and at 6,24 , and $72 \mathrm{~h}$ postnatal age according to presence (shaded columns, $n$ $=30$ ) or absence (unshaded columns, $n=44$ ) of PROM. Plasma levels of IFN- $\gamma$, IL-2, and TNF- $\alpha$ were increased in infants with PROM as compared those in infants without PROM at indicated postnatal ages $(* p<0.05, * * p<$ 0.01). Plasma levels of IL-6 increased from birth to $6 \mathrm{~h}$ of age in infants without PROM $(* p<0.05)$, with a subsequent decrease at $72 \mathrm{~h}\left({ }^{*} p<0.05\right)$. Level of IL- 6 was highest at birth and decreased at $72 \mathrm{~h}$ of age in infants with PROM $(* p<0.05)$. Medians and interquartile ranges are indicated. 


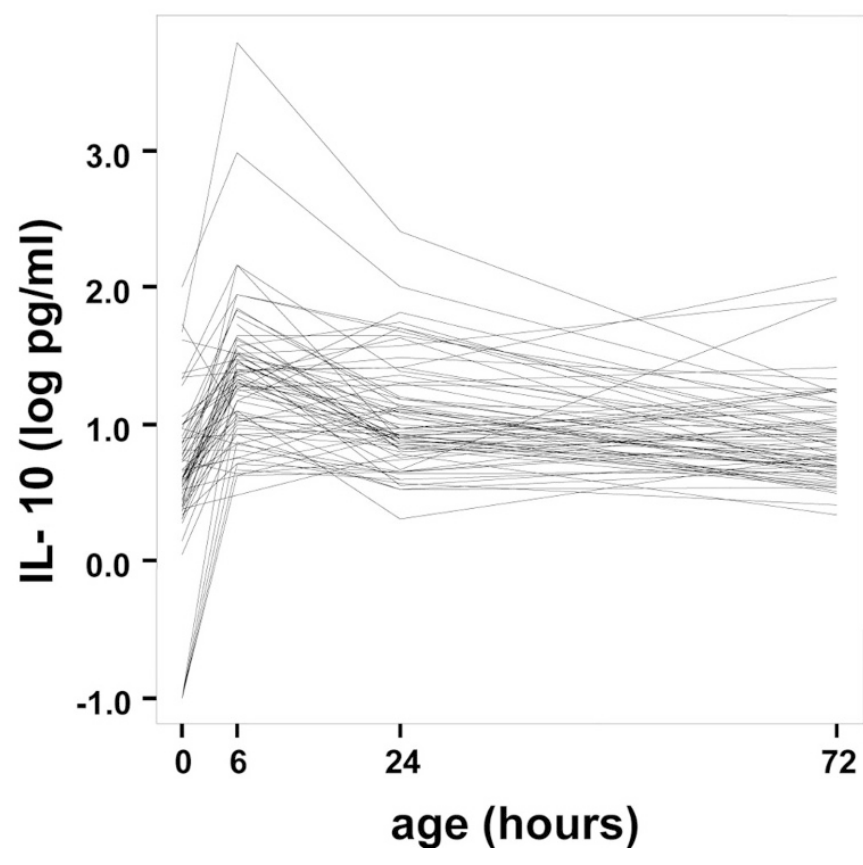

Figure 2. Individual plasma levels of IL-10 $(\log \mathrm{pg} / \mathrm{mL})$ at birth and at 6, 24, and $72 \mathrm{~h}$ postnatal age in preterm infants $(n=74)$.

Cytokines and arterial hypotension. Increases in AUC (IL-6) and in AUC (IL-8) were associated with a decrease in $\operatorname{MABP}(0-72 \mathrm{~h})(r=-0.37, p=0.002$, and $r=-0.39, p=$ 0.001 , respectively). A weaker inverse association was observed between AUC (IL-1 $\beta)$ and MABP $(r=-0.27, p=$ $0.027)$. No associations were present between $\operatorname{MABP}(0-72 \mathrm{~h})$ and AUC of any of the other cytokines. Levels of IL-6 at $6 \mathrm{~h}$ $(r=-0.36, p=0.003)$ and of IL-8 at $24 \mathrm{~h}(r=-0.46, p<$ $0.001)$ exhibited the strongest inverse association with average $\operatorname{MABP}(0-72 \mathrm{~h})$.

Increases in AUC (IL-6), AUC (IL-8), and AUC (IL-10) were associated with dopamine treatment during the first $72 \mathrm{~h}$, (OR, 11.8, 95\% CI, 2.6-53.5, $p<0.001$; OR, 17.9, 95\% CI, 2.5-129.9, $p<0.001$; and OR, 13.1, 95\% CI, 2.1-80.5, $p=$ 0.001 , respectively). Assessment of cytokine-levels at the respective sampling points showed that an increase in IL-6 at $6 \mathrm{~h}$ exhibited the strongest association with dopamine treatment ( $p$ $<0.001)$. Described associations remained significant after adjustment for GA and gender.

Infants receiving dopamine treatment $(0-72 \mathrm{~h})$ for arterial hypotension $(n=25)$ had a temporal pattern of IL-6 showing a marked increase in levels of IL- 6 from those in umbilical cord $58(2-342,646) \mathrm{pg} / \mathrm{mL}$ (median, range) to those at $6 \mathrm{~h}$ of age $923(19-866,057) \mathrm{pg} / \mathrm{mL}, p=0.011$, compared with unchanged levels in infants not requiring dopamine, 38 (1.5$8454)$ and 33 (4.5-634) $\mathrm{pg} / \mathrm{mL}$ respectively, $(p=0.186)$. Temporal course of IL-6 in infants with and without dopamine treatment for arterial hypotension is shown in Figure 3. A level of IL-6 $>184 \mathrm{pg} / \mathrm{mL}$ at $6 \mathrm{~h}$ was predictive of dopamine treatment $(0-72 \mathrm{~h})$, with a sensitivity of $67 \%$ and a specificity of $93 \%$ and with a positive predictive value of $84 \%$ and a negative predictive value of $84 \%$.

Cytokines and development of WMD and severe IVH. Increase in AUC (IFN- $\gamma$ ) was associated with development of

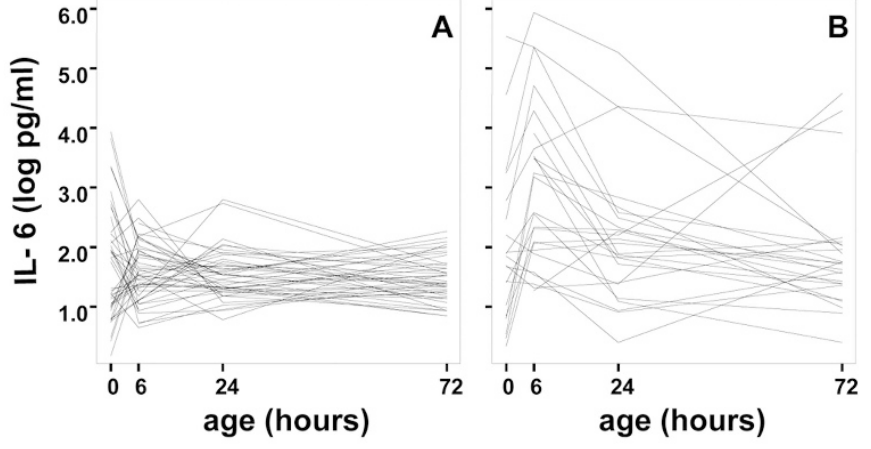

Figure 3. Individual plasma levels of IL-6 $(\log \mathrm{pg} / \mathrm{mL})$ at birth and at 6,24 , and $72 \mathrm{~h}$ postnatal age in infants requiring dopamine treatment for arterial hypotension $(n=25)$ and in those not requiring dopamine treatment $(n=49)$.

WMD (OR, 26.0. 95\% CI, 2.9-232.7, $p=0.002$ ). Levels of IFN- $\gamma$ at birth, 6, 24, and $72 \mathrm{~h}$ were increased in infants developing WMD compared with those without WMD: $p=$ $0.057, p=0.017, p=0.005$, and $p=0.04$ respectively. Median levels and distribution of IFN- $\gamma$ at sampling points in relation to development of WMD are given in Figure 4.

Temporal course of IL-6 in infants developing WMD was different compared with those requiring dopamine treatment for arterial hypotension. Median level (range) of IL-6 at birth was $124(5-342,648) \mathrm{pg} / \mathrm{mL}$ and showed a tendency to decrease at $6 \mathrm{~h}, 54(24-231,723) \mathrm{pg} / \mathrm{mL}(p=0.09)$, in infants developing WMD.

Increases in AUC (IL-6) and in AUC (IL-8) were associated with development of severe IVH (OR, 2.8, 95\% CI, 1.1-6.9, $p=0.023$ and $\mathrm{OR}, 13.2,95 \% \mathrm{CI}, 1.5-116.3, p=0.01$,

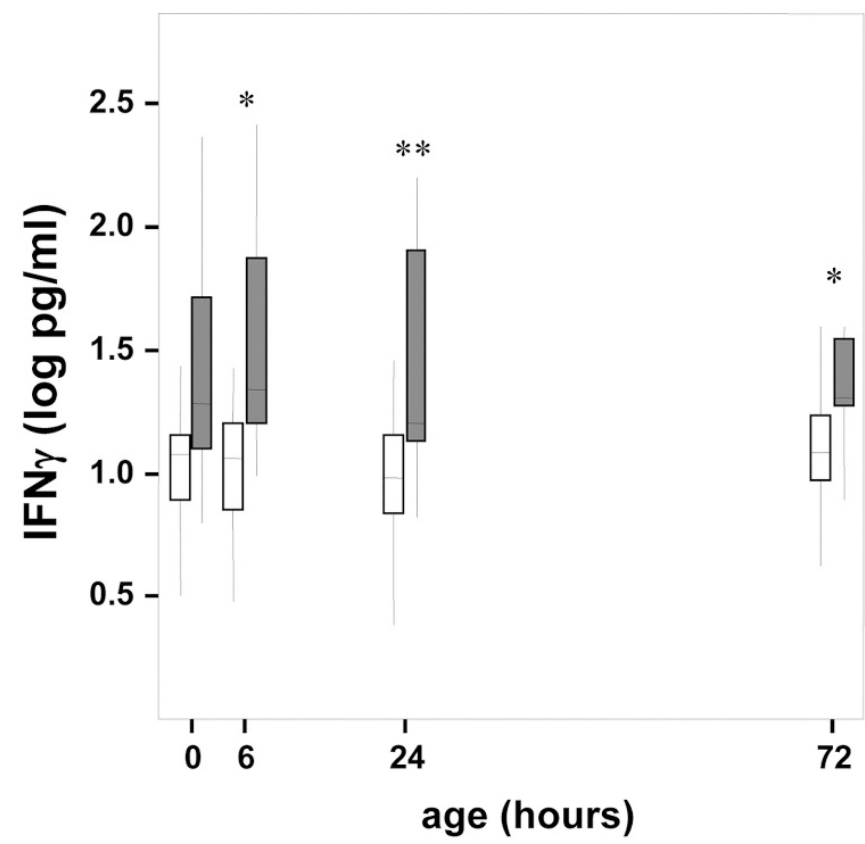

Figure 4. Plasma levels of IFN- $\gamma(\log \mathrm{pg} / \mathrm{mL})$ at birth and at 6,24 , and $72 \mathrm{~h}$ postnatal age in infants developing WMD (shaded columns, $n=8$ ) compared with infants without WMD (unshaded columns, $n=66$ ). Levels of IFN- $\gamma$ were increased in infants with WMD at indicated postnatal ages $\left({ }^{*} p<0.05\right.$, ${ }^{*} p<$ 0.01). Medians and interquartile ranges are indicated. 
respectively. No significant associations were observed between levels of IL- 6 and IL- 8 at the respective time points of sampling and development of severe IVH. Average MABP $(0-72 \mathrm{~h})$ was not associated with development of severe IVH or WMD in multivariate analyses and affected neither the relationship between AUC of IL- 6 and IL- 8 and severe IVH nor that between AUC (IFN- $\gamma$ ) and WMD.

\section{DISCUSSION}

Increased levels of circulating pro-inflammatory cytokines during the first $72 \mathrm{~h}$ of life were associated with arterial hypotension and with the development of brain damage as detected by ultrasound. Assessment of cytokine burden over time, as obtained by AUC from levels in umbilical cord and in postnatal blood up to $72 \mathrm{~h}$ postnatal age, showed the following: 1) increases in AUC of IL-6, IL-8, and IL-10 were associated with arterial hypotension; 2) increases in AUC of IL-6 and IL-8 were associated with severe IVH; and 3) PROM was associated with increased postnatal levels of IFN- $\gamma$, which in turn were associated with WMD. Infants with arterial hypotension requiring treatment had an early postnatal increase in IL-6, suggesting a recent induction of a systemic inflammatory response. Development of WMD, with increased postnatal levels of IFN- $\gamma$ at repeated time points, was neither associated with arterial hypotension nor with an early postnatal increase in IL-6.

Serial analysis of circulating cytokine levels at defined time points showed that levels of several cytokines change rapidly and by a large magnitude within the same subject, often by several orders. Subsequently, an assessment of circulating cytokine levels at a singular time point may not detect significant elevations and may therefore fail to detect relevant associations between inflammatory response and morbidity $(12,21)$. IL-6 and IL-8 exhibited similar changes over time, with levels being highest in umbilical cord or at $6 \mathrm{~h}$ postnatal age and subsequently decreasing and, in the vast majority of subjects, reaching lowest levels by $72 \mathrm{~h}$ of age. The modulatory cytokine IL-10 exhibited a characteristic temporal pattern with a postnatal peak at $6 \mathrm{~h}$ postnatal age and with a subsequent decrease up to $72 \mathrm{~h}$ of age in the majority of subjects. These changes over time suggest that systemic inflammatory activity during the first $3 \mathrm{~d}$ of age is initiated in utero in the majority of preterm subjects. This is an important finding and, although not unexpected, reinforces the hypothesis of fetal inflammatory response being associated with postnatal neurologic and respiratory morbidity.

Correlation analysis between plasma levels of different cytokines revealed several patterns of interest. Levels of the modulatory cytokine IL-10 were positively associated with those of the pro-inflammatory cytokines IL-6, IFN- $\gamma$, and IL-8, supporting an operating counter-regulatory mechanism during early postnatal age in preterm infants. Serial analysis of IL-10 revealed an extremely homogenous temporal profile with a distinct peak at $6 \mathrm{~h}$ occurring in almost all infants. IL-10 is produced by Th 2 cells, B cells, and macrophages as well as by several placental tissues and has a down-regulatory effect on production of pro-inflammatory cytokines (22). Parturition in- creases placental IL-10 production in response to proinflammatory stimuli, and catecholamines have been shown to be effective stimulants for IL-10 secretion in humans $(22,23)$. Although levels of IL-10 were positively correlated to those of several pro-inflammatory cytokines, suggesting that IL-10 increased as a counter-regulatory mechanism, the observed early postnatal increase in IL-10 suggests an effect of birth per se. The influence of early invasive procedures such as umbilical catheterization on systemic inflammatory response is unknown but may hypothetically have affected levels of circulating cytokines at $6 \mathrm{~h}$ of age.

Levels of TNF- $\alpha$ and IL- $1 \beta$ were frequently nondetectable at all studied time points. Yanowitz et al. (7) detected TNF- $\alpha$ in cord blood in only $20 \%$ of the preterm infants. Lipopolysaccharide stimulation in adults results in an earlier dosedependent peak for TNF- $\alpha$ than for IL-6. To some extent, TNF- $\alpha$ triggers the release of IL-6 (11). IL-6 has a suppressive effect on the production of both TNF- $\alpha$ and IL-1 $\beta$ (24). This suggests that levels of TNF- $\alpha$ and IL- $1 \beta$ may have peaked before birth, thereby escaping detection in postnatal blood and in turn disabling a meaningful evaluation of their relation to morbidity.

Inflammatory response and arterial hypotension. Increased circulating levels of IL- 6 and IL-8 were associated with a decrease in MABP, with levels of IL- 6 at $6 \mathrm{~h}$ postnatal age being a good predictor of requirement of dopamine treatment for arterial hypotension. These findings are similar to those of Yanowitz et al. (7), who showed an association between IL-6 in umbilical cord blood, histologic chorioamnionitis, and a decrease in MABP at 4-6 h postnatal age. The temporal pattern of IL-6 revealed that circulatory impairment was prevalent in those infants exhibiting an increase in IL-6 during the first $6 \mathrm{~h}$ of life. The described early postnatal increase of IL-6, often by several orders, with a peak at $6 \mathrm{~h}$, suggests a recent induction of the inflammatory response. Several monoamines, including dopamine, have been shown to increase synthesis of IL-6, thus inferring that exogenous dopamine may have caused the described increase in IL-6 $(23,25)$. This seems less likely as dopamine treatment initiated before $6 \mathrm{~h}$ of age was not associated with higher levels of IL-6 at $6 \mathrm{~h}$ compared with levels in infants receiving dopamine at a later age. Similarly, dopamine treatment initiated after 6 or $24 \mathrm{~h}$ of age did not result in a subsequent increase in IL-6.

Increased systemic pro-inflammatory activity may affect several mechanisms leading to circulatory impairment. Proinflammatory cytokines cause an increased production of NO by up-regulating inducible NO-synthase, resulting in decreased vessel wall tone (26). Up-regulation of adhesion molecules with increased adhesion of leukocytes to the endothelial wall causes a release of proteases and reactive oxygen species (27). Increased reactive oxygen species generation is present during inflammation and can cause an inactivation of catecholamines (28). Activation of all these mechanisms leads to increased vascular permeability and peripheral vasodilation with a decrease in vascular resistance. IL-6 as well as IL-8 appear to be present in high concentrations and with a relatively long half-life during the inflammatory response in the preterm infant. IL-6 is readily detected and therefore a sensitive circula- 
tory marker of timing and magnitude of the inflammatory response.

Arterial hypotension has been considered a risk factor for cerebral injury inasmuch as the germinal matrix and cerebral white matter are highly vulnerable to hypoperfusion due to the watershed vascular supply in these areas. We found no association between average MABP during the first $72 \mathrm{~h}$ and either IVH or WMD in our study. We did, however, find an association between increases in levels of IL- 6 and IL- 8 and development of severe IVH, as did Heep et al. (8). Other studies suggests that low cerebral blood flow rather than blood pressure is associated with brain damage and that cerebral blood flow can be maintained in spite of low MABP $(29,30)$. Lipopolysaccharide-induced inflammation in fetal sheep has shown resulting fetal hypoxia due to a reduction of placental blood flow with a subsequent reduction in cerebral oxygen delivery (31). No study has, to our knowledge, shown an actual decrease in cerebral blood flow due to inflammation in the absence of induced asphyxia.

Inflammatory response and WMD. Circulating levels of IFN- $\gamma$ were increased in infants developing WMD. The major pathologic feature of WMD or PVL is a chronic disturbance of myelination, which suggests that the oligodendrocyte cell lineage is affected. WMD usually develops between 24 and $32 \mathrm{wk}$ gestation, and during this period the human parietal white matter is populated mostly by oligodendrocyte progenitors. Several in vitro studies have shown that IFN- $\gamma$ reduces proliferation and viability of oligodendroglial cells and their progenitors, the preoligodendrocytes, appear to be more sensitive than the more differentiated cells (32). This would appear to support our finding of elevated circulating IFN- $\gamma$ being actually implicated in the pathogenesis of WMD. The findings of increased circulatory levels of these cytokines does not necessarily imply increased levels within the CNS. However, administration of endotoxin in fetal sheep seems to increase blood-brain barrier permeability and cytokines in the circulation have been shown to cross the blood-brain barrier, although it is unknown whether this passage is sufficient to cause an inflammatory responses within the brain $(33,34)$. Intravenous administration of lipopolysaccharide in vivo results in neuropathological lesions similar to those in PVL (35). Lipopolysaccharide activates circulating immune cells and microglia through the tolllike receptor resulting in synthesis of pro-inflammatory cytokines and reactive oxygen species. Both of these substance groups have known damaging effects to oligodendrocyte progenitors (36).

The increased circulating levels of IFN- $\gamma$ observed in infants with WMD may reflect elevated levels of the same cytokine within the brain. A common stimulus, one of various possible microbial compounds, may initiate activation of immune cells within the vascular system as well as the microglia within the brain resulting in elevated levels of IFN- $\gamma$ in both compartments. Increased circulatory levels of IFN- $\gamma$ may also increase blood-brain barrier permeability, resulting in infiltration of circulatory immune cells to the brain with subsequent increased local pro-inflammatory activity causing damage to oligodendrocyte progenitors. Very recently, Bell et al. (37) showed a 20 -fold increase in levels of IFN- $\gamma$ protein in the fetal brain in a rat model with lipopolysaccharide-induced intrauterine inflammation. Expression of IFN- $\gamma$ protein has also been shown to be increased in macrophages and activated astrocytes in human perinatal brains with PVL (38). The same study showed that the IFN- $\gamma$ receptor was expressed in human premyelinating oligodendrocytes, which suggested a potential for IFN- $\gamma$ induced toxicity to those cells via receptor-mediated mechanisms. Those results suggest that our finding of increased circulating levels of IFN- $\gamma$ in preterm infants who developed WMD may reflect increased levels of the same cytokine within the brain and more importantly, that IFN- $\gamma$ may be implicated in the pathogenesis of WMD.

Infants to mothers with PROM had increased circulating levels of IFN- $\gamma$, IL-2, and TNF- $\alpha$, all three cytokines belonging to the Th1 subset of T-helper cells. A Th1 predominance with increased levels of IFN- $\gamma$ in cord blood and in maternal blood after PROM has been observed previously $(16,17)$. Infants to mothers with PROM have also been suggested to be at an increased risk for development of cystic PVL $(6,39)$. Our finding of increased levels of IFN- $\gamma$ in infants delivered after PROM and in infants developing WMD supports the association between induced antenatal inflammation and WMD and proposes a possible mechanistic pathway. In the absence of placental histology, we chose PROM as a marker of antenatal inflammation because it has been shown as a condition with a high prevalence of intra-amniotic inflammation and infection (15).

We found positive correlations between circulatory levels of IFN- $\gamma$, IL-10, and IL-12 within the whole study group indicating that levels of the respective cytokines were frequently increased simultaneously. IL-10 has modulating effects on production of IFN- $\gamma$ and inhibits IFN- $\gamma$ mediated oligodendroglial death by suppressing inducible nitric oxide synthase (iNOS) thus inhibiting NO-production (40). IL-12, on the other hand, can induce IFN- $\gamma$ production (14). We were unable to show any interaction between IFN- $\gamma$, IL-10, and IL-12 in the development of WMD when applying multivariate analysis. Such analysis is, however, limited by the relatively small number of infants developing WMD in the present study.

The pro-inflammatory cytokine IFN- $\gamma$ is a member of the Th1 cytokine family, as opposed to IL-6 and IL-10, which belong to the Th2 cytokine pattern. Increased synthesis of cytokines belonging to either the Th1 or the Th2 pattern has been ascribed to differences in the inducing compound (14). The finding of increased IFN- $\gamma$ in association with WMD as opposed to that of IL-6 in association with IVH and circulatory impairment may depend on differences in the microbial compounds responsible for inducing the inflammatory response.

The described early postnatal increase in circulatory levels of IL-6 associated with arterial hypotension was not present in infants developing WMD. On the contrary, levels of IL-6 were relatively high in the umbilical cord, with a tendency to decrease at $6 \mathrm{~h}$ in infants developing WMD. This observation lends further support to our hypothesis that induction of inflammatory response probably took place earlier in infants developing WMD than in those exhibiting an immediate rise in IL-6 after birth and with postnatal arterial hypotension requiring treatment. 
We suggest that an induction of inflammatory response in utero that is not followed by delivery presents a greater risk for development of WMD than an inflammatory response leading to immediate delivery. This is supported by a recent study showing that T-cell activation in umbilical cord blood reflecting in utero initiation of inflammatory response was followed by early cerebral damage as detected by magnetic resonance at a median age of $2 \mathrm{~d}(9)$.

In conclusion, we found that increased postnatal circulating levels of IFN- $\gamma$ were associated with PROM and with development of WMD, whereas increased levels of IL-6 and IL-8 were associated with severe IVH and arterial hypotension. Based on our data and those of previous studies, we hypothesize two mechanistic pathways leading to different entities of impairment. Early induction of inflammation causing a preferable activation of Th1 cytokines, such as IFN- $\gamma$, TNF- $\alpha$, and IL-2, leads to disturbed oligodendrocyte viability with an increased risk for subsequent development of WMD. Inflammation followed by an immediate delivery, with an early postnatal increase in IL-6 and IL-8, leads to circulatory impairment and subsequent risk for IVH. Thus, timing and type of induction of inflammatory response appear of importance for subsequent morbidity.

Acknowledgments. The authors thank Jeanette Arvastsson for technical help with CBA and flow cytometry, Prof. Vineta Fellman for constructive comments, and Per-Erik Isberg for advice on statistics.

\section{REFERENCES}

1. Dammann O, Leviton A 1997 Maternal intrauterine infection, cytokines, and brain damage in the preterm newborn. Pediatr Res 42:1-8

2. Gomez R, Romero R, Ghezzi F, Yoon BH, Mazor M, Berry SM 1998 The fetal inflammatory response syndrome. Am J Obstet Gynecol 179:194-202

3. De Felice C, Toti P, Laurini RN, Stumpo M, Picciolini E, Todros T, Tanganelli P, Buonocore G, Bracci R 2001 Early neonatal brain injury in histologic chorioamnionitis. J Pediatr 138:101-104

4. Dammann O, Allred EN, Veelken N 1998 Increased risk of spastic diplegia among very low birth weight children after preterm labor or prelabor rupture of membranes. J Pediatr 132:531-535

5. Yoon BH, Romero R, Park JS, Kim CJ, Kim SH, Choi JH, Han TR 2000 Fetal exposure to an intra-amniotic inflammation and the development of cerebral palsy at the age of three years. Am J Obstet Gynecol 182:675-681

6. Zupan V, Gonzalez P, Lacaze-Masmonteil T, Boithias C, d'Allest AM, Dehan M, Gabilan JC 1996 Periventricular leukomalacia: risk factors revisited. Dev Med Child Neurol 38:1061-1067

7. Yanowitz TD, Jordan JA, Gilmour CH, Towbin R, Bowen A, Roberts JM, Brozanski BS 2002 Hemodynamic disturbances in premature infants born after chorioamnionitis: association with cord blood cytokine concentrations. Pediatr Res 51:310-316

8. Heep A, Behrendt D, Nitsch P, Fimmers R, Bartmann P, Dembinski J 2003 Increased serum levels of interleukin 6 are associated with severe intraventricular haemorrhage in extremely premature infants. Arch Dis Child Fetal Neonatal Ed 88:F501-F504

9. Duggan PJ, Maalouf EF, Watts TL, Sullivan MH, Counsell SJ, Allsop J, Al-Nakib L, Rutherford MA, Battin M, Roberts I, Edwards AD 2001 Intrauterine T-cell activation and increased proinflammatory cytokine concentrations in preterm infants with cerebral lesions. Lancet 358:1699-1700

10. Nelson KB, Grether JK, Dambrosia JM, Walsh E, Kohler S, Satyanarayana G, Nelson PG, Dickens BF, Phillips TM 2003 Neonatal cytokines and cerebral palsy in very preterm infants. Pediatr Res 53:600-607

11. Vedder H, Schreiber W, Yassouridis A, Gudewill S, Galanos C, Pollmacher T 1999 Dose-dependence of bacterial lipopolysaccharide (LPS) effects on peak response and time course of the immune-endocrine host response in humans. Inflamm Res 48:67-74
12. Hagberg H 2003 No correlation between cerebral palsy and cytokines in postnatal blood of preterms. Pediatr Res 53:544-545

13. Dammann O, Phillips TM, Allred EN, O'Shea TM, Paneth N, Van Marter LJ, Bose C, Ehrenkranz RA, Bednarek FJ, Naples M, Leviton A; Elgan Study Investigators 2001 Mediators of fetal inflammation in extremely low gestational age newborns. Cytokine 13:234-239

14. Romagnani S 2000 T-cell subsets (Th1 versus Th2). Ann Allergy Asthma Immunol 85:9-18; quiz 18:21

15. Shim SS, Romero R, Hong JS, Park CW, Jun JK, Kim BI, Yoon BH 2004 Clinical significance of intra-amniotic inflammation in patients with preterm premature rupture of membranes. Am J Obstet Gynecol 191:1339-1345

16. Yoneyama Y, Suzuki S, Sawa R, Otsubo Y, Miura A, Kuwabara Y, Ishino H, Kiyokawa Y, Doi D, Yoneyama K, Araki T 2003 Changes in the proportion of T helper 1 and $\mathrm{T}$ helper 2 cells in cord blood after premature rupture of membranes. Arch Gynecol Obstet 267:217-220

17. Raghupathy R, Makhseed M, El-Shazly S, Azizieh F, Farhat R, Ashkanani L 2001 Cytokine patterns in maternal blood after premature rupture of membranes. Obstet Gynecol 98:122-126

18. de Vries LS, Eken P, Dubowitz LM 1992 The spectrum of leukomalacia using cranial ultrasound. Behav Brain Res 49:1-6

19. Marsal K, Persson PH, Larsen T, Lilja H, Selbing A, Sultan B 1996 Intrauterine growth curves based on ultrasonically estimated foetal weights. Acta Paediatr 85:843-848

20. Altman DG 1991 Practical Statistics for Medical Research. Chapman and Hall, London, pp xii, 611

21. Harding D, Dhamrait S, Millar A, Humphries S, Marlow N, Whitelaw A, Montgomery H 2003 Is interleukin-6-174 genotype associated with the development of septicemia in preterm infants? Pediatrics 112:800-803

22. Simpson KL, Keelan JA, Mitchell MD 1998 Labor-associated changes in interleukin-10 production and its regulation by immunomodulators in human choriodecidua. J Clin Endocrinol Metab 83:4332-4337

23. Uusaro A, Russell JA 2000 Could anti-inflammatory actions of catecholamines explain the possible beneficial effects of supranormal oxygen delivery in critically ill surgical patients? Intensive Care Med 26:299-304

24. Schindler R, Mancilla J, Endres S, Ghorbani R, Clark SC, Dinarello CA 1990 Correlations and interactions in the production of interleukin-6 (IL-6), IL-1, and tumor necrosis factor (TNF) in human blood mononuclear cells: IL-6 suppresses IL-1 and TNF. Blood 75:40-47

25. Ritchie PK, Ashby M, Knight HH, Judd AM 1996 Dopamine increases interleukin 6 release and inhibits tumor necrosis factor release from rat adrenal zona glomerulosa cells in vitro. Eur J Endocrinol 134:610-616

26. Cohen J 2002 The immunopathogenesis of sepsis. Nature 420:885-891

27. Colman RW, Schmaier AH 1997 Contact system: a vascular biology modulator with anticoagulant, profibrinolytic, antiadhesive, and proinflammatory attributes. Blood 90:3819-3843

28. Pleiner J, Mittermayer F, Schaller G, Marsik C, MacAllister RJ, Wolzt M 2003 Inflammation-induced vasoconstrictor hyporeactivity is caused by oxidative stress. J Am Coll Cardiol 42:1656-1662

29. Tyszczuk L, Meek J, Elwell C, Wyatt JS 1998 Cerebral blood flow is independent of mean arterial blood pressure in preterm infants undergoing intensive care. Pediatrics 102:337-341

30. Meek JH, Tyszczuk L, Elwell CE, Wyatt JS 1999 Low cerebral blood flow is a risk factor for severe intraventricular haemorrhage. Arch Dis Child Fetal Neonatal Ed 81:F15-F18

31. Dalitz P, Harding R, Rees SM, Cock ML 2003 Prolonged reductions in placental blood flow and cerebral oxygen delivery in preterm fetal sheep exposed to endotoxin: possible factors in white matter injury after acute infection. J Soc Gynecol Investig 10:283-290

32. Baerwald KD, Popko B 1998 Developing and mature oligodendrocytes respond differently to the immune cytokine interferon-gamma. J Neurosci Res 52:230-239

33. Yan E, Castillo-Melendez M, Nicholls T, Hirst J, Walker D 2004 Cerebrovascular responses in the fetal sheep brain to low-dose endotoxin. Pediatr Res 55:855-863

34. Banks WA, Kastin AJ, Durham DA 1989 Bidirectional transport of interleukin-1 alpha across the blood-brain barrier. Brain Res Bull 23:433-437

35. Duncan JR, Cock ML, Scheerlinck JP, Westcott KT, McLean C, Harding R, Rees SM 2002 White matter injury after repeated endotoxin exposure in the preterm ovine fetus. Pediatr Res 52:941-949

36. Volpe JJ 2001 Neurobiology of periventricular leukomalacia in the premature infant Pediatr Res 50:553-562

37. Bell MJ, Hallenbeck JM, Gallo V 2004 Determining the fetal inflammatory response in an experimental model of intrauterine inflammation in rats. Pediatr Res 56:541-546

38. Folkerth RD, Keefe RJ, Haynes RL, Trachtenberg FL, Volpe JJ, Kinney HC 2004 Interferon-gamma expression in periventricular leukomalacia in the human brain. Brain Pathol 14:265-274

39. Perlman JM, Risser R, Broyles RS 1996 Bilateral cystic periventricular leukomalacia in the premature infant: associated risk factors. Pediatrics 97:822-827

40. Molina-Holgado E, Vela JM, Arevalo-Martin A, Guaza C 2001 LPS/IFN-gamma cytotoxicity in oligodendroglial cells: role of nitric oxide and protection by the anti-inflammatory cytokine IL-10. Eur J Neurosci 13:493-502 